\title{
Work in Progress: \#FunTimesWithTheTA - A Series of Fun Supplementary Lessons for Introductory Level Biomedical Instrumentation Students (Part II)
}

\author{
Mr. Orlando Sanguinette Hoilett, Purdue University, West Lafayette (College of Engineering)
}

Orlando is a PhD student in Biomedical Engineering at Purdue University. Orlando graduated from Vanderbilt University in 2014 with a Bachelor of Engineering in Biomedical Engineering. His academic interests involve developing wearable sensors for mobile health platforms and developing engaging engineering design activities for undergraduate students. He has several years of experience with analog circuit design and embedded systems. His previous work includes developing an impedance analyzer for a microfluidic blood-brain barrier model. On the education side, he has developed a number of lab activities for first year and junior level Biomedical Engineering students. Aside from his academic pursuits, he is an avid electronics hobbyist and tinkerer. He spends most of his free time developing open source hardware for students, educators, and makers alike.

\section{Asem Farooq Aboelzahab, Purdue University, West Lafayette (College of Engineering)}

Asem Aboelzahab is the Lab and Assessment Coordinator in the Weldon School of Biomedical Engineering at Purdue University. He has been at Purdue since 2014. He instructs/coordinates undergraduate labs including Bioinstrumentation, Biotransport, and Capstone Senior Design. He also serves as the school's ABET coordinator. Asem received his BS and MS degrees in Bioengineering from the University of Toledo in Toledo, Ohio.

\section{Dr. Erica Lott, Purdue University, West Lafayette (College of Engineering)}

Dr. Erica Lott is an Instructional Developer at the Center for Instructional Excellence at Purdue University in West Lafayette, IN. She earned her Ph.D in College Science Teaching specializing in Earth Sciences from Syracuse University. Her research interests include, but are not limited to: learners' understanding and representation of physical phenomena, course transformations and their implications for teaching and learning, discourse analysis of scientific classroom talk, and science teacher education.

\section{Dr. Jacqueline Callihan Linnes, Purdue University}

Dr. Jacqueline Callihan Linnes is an assistant professor of Biomedical Engineering at Purdue University. She earned her Ph.D. in Bioengineering and certificate in Global Health from the University of Washington. She was a Fogarty engineering fellow in collaboration with Brigham and Women's Hospital and the Little Devices laboratory at MIT before moving to Boston University's Biomedical Engineering department where she received a NIH NRSA postdoctoral fellowship to develop molecular diagnostics for point-of-care pathogen detection. Dr. Linnes's current research bridges innovations in basic science and translational diagnostic techniques in order to develop non-invasive, rapid detection technologies that efficiently diagnose and monitor diseases at the point of care. Her teaching focuses experiential learning and co-creation of technologies via user-centered design and evaluation. 


\section{\#FunTimesWithTheTA-A Series of Fun, Supplementary Lessons for Introductory Level Biomedical Instrumentation Students (Part II) (Work-in-Progress)}

\#FunTimesWithTheTA is a series of low-risk, low-stress, supplementary, active-learning lessons [1] developed for introductory biomedical instrumentation students. The purpose of these supplementary lessons is to reinforce the principles taught in the normally-scheduled course [2], [3] as well as introduce students to new design concepts that are applicable to them as budding engineers. We have observed that under the rigor of the engineering curriculum, students often lose sight of the practical benefits of the engineering principles taught in their coursework. We hypothesize that by providing students with these supplementary opportunities, students will be encouraged in their coursework [4] as well as learn a few new concepts.

The core learning objectives of the normally-scheduled course include students gaining competency using electrical test equipment, such as oscilloscopes and function generators, as well as developing fundamental analog circuit design skills such as first-order filter design and implementation. As a result, \#FunTimesWithTheTA focused on lessons that required students to utilize electrical test equipment and design filters for various applications. These applications included measuring biopotential signals, such as an electrocardiogram or electrooculogram, and manipulating an audio signal from an MP3 player to create a "Do-It-Yourself" sound level meter. Furthermore, \#FunTimesWithTheTA introduced students to applications of sensors in biomedical circuits, which expand past the scope of the normally-scheduled course.

As a second iteration of our study, we implemented a simple survey. We asked students to reflect on their experience with the supplementary lessons and compare elements of this experience to the environment of the normally-scheduled course. The survey is designed around the basic psychological needs of the students, namely perceptions of autonomy and competency [5], [6]. We aimed to evaluate whether or not students felt comfortable in the new learning environment and whether or not \#F unTimesWithTheTA Super Simple EXG Circuit Rev A

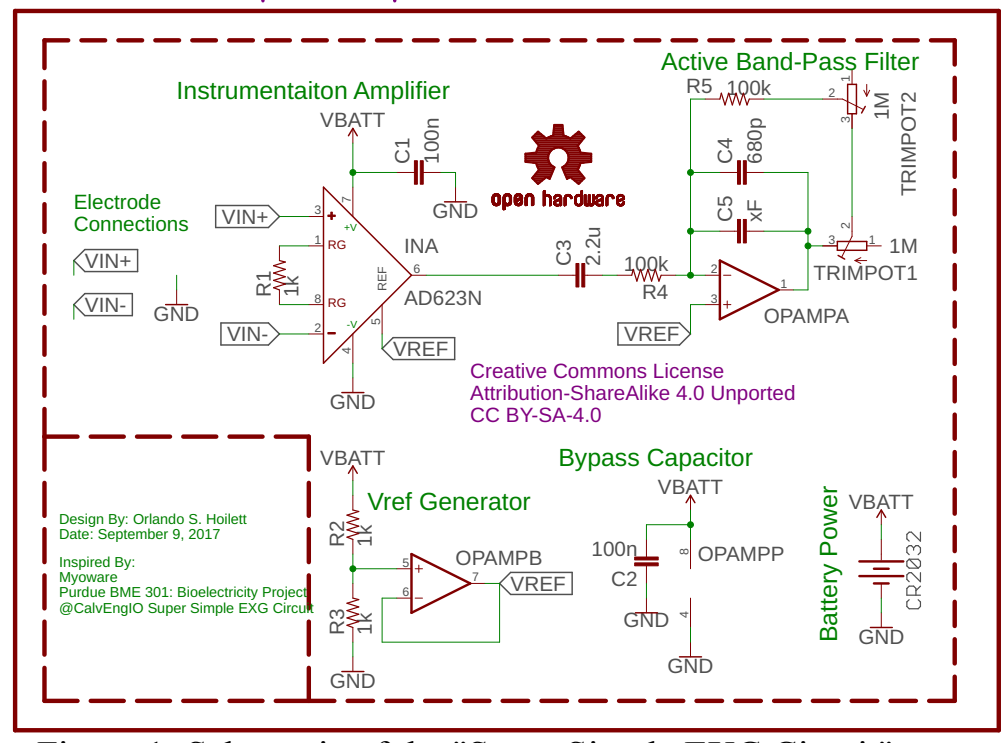

Figure 1: Schematic of the "Super Simple EXG Circuit" used to measure electrocardiogram (ECG), electrooculogram (EOG), and electromyogram (EMG) signals. their circuit design skills were improving as a result of attending the lessons.

\section{Methods}

We have previously reported on our pilot in an earlier publication [7], so the methods will be described briefly here. The lessons take place during the latter half of the normally-scheduled 
office hours. Students self-select into groups in order to complete different parts of the circuit. Though students will not always have a choice of teams in their professional careers, research is clear that providing students autonomy in their learning environment fosters collaboration and student-driven learning [8]-[10]. After completing their chosen subcomponents, students then combined their parts in order to create the completed circuit. Attendance is voluntary and is not factored into students' grades. The lessons are developed by the Graduate Teaching Assistant (GTA) and are complete with step-by-step instructions. An example circuit is shown in Figure 1. The remaining lessons can be found in an online repository [11].

Surveys were distributed to students at the end of the semester. Survey questions were paired such that we could compare students' assessment of \#FunTimesWithTheTA with that of the normal course. We separated student responses based on which element of the student experience we were analyzing, namely competency (Table 1) and autonomy (Table 2). Six questions were related to autonomy (3 questions asked about \#FunTimesWithTheTA, while 3 questions asked about the regular course) and twelve questions were related to competency ( 6 questions asked about \#FunTimesWithTheTA, while 6 questions asked about the regular course).

Furthermore, we categorized student responses into two groups: 1) students who attended 2 or more sessions of \#FunTimesWithTheTA and 2) students who attended only 1 session. We tallied the number of times students selected either "Strongly Agree", "Agree", "Somewhat agree", "Neither agree nor disagree", "Somewhat disagree", "Disagree", "Strongly Disagree" in the survey. Survey data was encoded such that a "7" represents the most positive response to a question, while a " 1 " represents the most negative response. This was done as one question asked students if they felt pressured in the course and in \#FunTimesWithTheTA. A positive response to this question would be "Strongly Disagree", while a positive response to most other questions would be "Strongly Agree".

We then calculated a weighted average score on a Likert scale from 1 (lowest) to 7 (highest) for each set of questions (competency and autonomy) and for each group of students (those who attended 2 or more sessions of \#FunTimesWithTheTA and those who attended only 1 session). To calculate standard deviation, we averaged each student's score for competency and autonomy. We then calculated variation within each group of students (attended 1 session vs. attended 2 or more sessions, \#FunTimesWithTheTA vs. Course). We cannot list all questions from the survey in this report, however, we include a few sample questions below:

Sample Q1. I have been able to learn interesting new skills in this course Sample Q2. I have been able to learn interesting new skills in \#FunTimesWithTheTA

Sample Q3. I am free to express my ideas and opinions in this course

Sample Q4. I am free to express my ideas and opinions in \#FunTimesWithTheTA

\section{Results}

Ten students attended our first session and at least 2 students attended each of the remaining sessions. One student attended 5 different sessions and another student attended 4. Our first session had the advantage of occurring early in the semester when the students' overall workloads were light. As the semester progressed, less students attended the sessions, which was expected. Of the 84 students enrolled in the course, 6 students responded to our survey. Though we did not obtain enough survey responses to provide statistical significance to the results for the entire class, from the students who responded to the survey, we observe that the lessons were 
generally favorable. In some cases, students responded more favorably to the lessons than to the regular course. Specifically, we observed statistical significance in reported degree of autonomy in \#FunTimesWithTheTA compared to the course. Furthermore, the two students who attended 2 or more sessions reported much more favorable outcomes from attending \#FunTimesWithTheTA than from the normally-schedule course with regards to both competency and autonomy. We observe higher average scores reported for both autonomy and competency for \#FunTimesWithTheTA than for the normal course. We postulate that with an increased sample size, we may observe more statistical significance in our dataset.

Table 1: Weighted Scores (1-lowest, 7-highest) Showing Outcomes from \#FunTimesWithTheTA and from the Normal Course with Respect to Competency and Autonomy

\begin{tabular}{|l|c|c|c|}
\hline COMPETENCY & Attended 1 Session & Attended 2+ Sessions & Total \\
\hline \#FunTimesWithTheTA & $5.96(0.417)$ & $6.92(0.118)$ & $6.28(0.593)$ \\
\hline Course & $6.38(0.210)$ & $5.58(0.589)$ & $6.11(0.513)$ \\
\hline \multicolumn{4}{|l|}{} \\
\hline AUTONOMY & Attended 1 Session & Attended 2+ Sessions* & Total $^{*}$ \\
\hline \#FunTimesWithTheTA & $5.75(0.631)$ & $6.83(0.236)$ & $6.11(0.750)$ \\
\hline Course & $5.00(0.609)$ & $4.67(0.471)$ & $4.89(0.544)$ \\
\hline
\end{tabular}

Responses for each student were averaged. Standard deviation was computed across the average values for each student within a group $(n=4$ : Attended 1 Session and $n=2$ : Attended 2+ Sessions $)$. *indicates $p$ $<0.05$ Student's paired t-Test, with two-tailed distribution.

\section{Informal Observations}

The level of curiosity and enthusiasm displayed by the students was very high. Students were inquisitive and very excited to see what we were building in the sessions. In many cases, students who attended the lessons showcased what they built to other students around the building. The GTA often overheard students discussing the lessons throughout the week. Students who attended \#FunTimesWithTheTA commented that the lessons were fun and regularly asked when the next session would be. We propose that \#FunTimesWithTheTA can help develop rapport between the instructor and the students by providing a more relaxed setting than the normallyscheduled office hours. This may lead to better student outcomes as others have shown in their study [9]. The GTA noted that students who attended \#FunTimesWithTheTA felt very comfortable approaching him for other career and research opportunities and were more comfortable asking questions during the normally-scheduled class time. The GTA also noted several instances where students finally "seemed to get it" after repeated interactions during \#FunTimesWithTheTA. For example, during normal course activities, students frequently used statements such as (paraphrased), "Like what we did in \#FunTimesWithTheTA" when describing their approach to the course activities.

\section{Future Directions}

We will continue with Part III of our study in the upcoming fall semester with another cohort of students. One of our major goals is to increase student attendance. Attendance this year was lower than we would have liked, though higher than the previous year [7], and did not provide statistical significance to our results for the entire class. We suspect, however, that lack of attendance is simply due to demands of prior commitments as opposed to lack of interest. In the survey, we asked students about major barriers preventing them from attending more of the 
lessons. All students cited prior commitments, either employment or coursework, as being barriers to attending.

In order to encourage student participation, we propose rewarding extra credit to students who attend the lessons. Previous literature has shown that offering modest extra credit improves student motivation, learning, and attendance in university courses [12], [13]. Furthermore, now that we have completed two years of the pilot study, we would like to ask students who have taken the lessons in previous cohorts about how the lessons may have impacted them in their future coursework, particularly their design courses. During informal conversations with students, at least two have mentioned to the GTA how their outlook on design has been influenced by \#FunTimesWithTheTA. Students noted that they took design principles learned in \#FunTimesWithTheTA and applied them to their senior design projects. Given the small size of our initial subject pool, we find these positive comments very encouraging.

Acknowledgements: We would like to thank Ms. Attiyya Houston for designing our logo.

\section{References}

[1] C. C. Bonwell and J. A. Eison, Active Learning: Creating Excitement in the Classroom. 1991 ASHE-ERIC Higher Education Reports. ERIC Clearinghouse on Higher Education, 1991.

[2] C. L. Dym, A. M. Agogino, O. Eris, D. D. Frey, and L. J. Leifer, "Engineering design thinking, teaching, and learning," J. Eng. Educ., vol. 94, no. 1, pp. 103-120, 2005.

[3] D. Jonassen, J. Strobel, and C. B. Lee, "Everyday Problem Solving in Engineering: Lessons for Engineering Educators," J. Eng. Educ., vol. 95, no. 2, pp. 139-151, Apr. 2006.

[4] P. Fonseca, P. Pedreiras, P. Cabral, J. N. Matos, B. Cunha, and F. Silva, "Motivating first year students for an engineering degree," in 2016 2nd International Conference of the Portuguese Society for Engineering Education (CISPEE), 2016, pp. 1-7.

[5] T. Knapp, B. Fisher, and C. Levesque-Bristol, "Service-Learning's Impact on College Students' Commitment to Future Civic Engagement, Self-Efficacy, and Social Empowerment," J. Community Pract., vol. 18, no. 2-3, pp. 233-251, Aug. 2010.

[6] C. Levesque-Bristol, T. D. Knapp, and B. J. Fisher, "The Effectiveness of ServiceLearning: It's Not Always what you Think," J. Exp. Educ., vol. 33, no. 3, pp. 208-224, Mar. 2011.

[7] O. S. Hoilett, A. F. Aboelzahab, E. A. Layow, J. C. Linnes, and C. H. Lee, "Board \# 8 :\#FunTimesWithTheTA-A Series of Fun Supplementary Lessons for Introductory Level Biomedical Instrumentation Students (Work in Progress)," presented at the 2017 ASEE Annual Conference \& Exposition, 2017.

[8] J. Sibthorp, K. Paisley, J. Gookin, and N. Furman, “The Pedagogic Value of Student Autonomy in Adventure Education," J. Exp. Educ., vol. 31, no. 2, pp. 136-151, Nov. 2008.

[9] Black Aaron E. and Deci Edward L., "The effects of instructors' autonomy support and students' autonomous motivation on learning organic chemistry: A self- determination theory perspective,"Sci. Educ., vol. 84, no. 6, pp. 740-756, Oct. 2000.

[10] W. Drexler, "The networked student model for construction of personal learning environments: Balancing teacher control and student autonomy," Australas. J. Educ. Technol., vol. 26, no. 3, May 2010.

[11] O. Hoilett, "\#FunTimesWithTheTA," GitHub. [Online]. Available: https://github.com/FunTimesWithTheTA. [Accessed: 30-Apr-2017]. 
[12] L. M. Padilla-Walker, "The Impact of Daily Extra Credit Quizzes on Exam Performance," Teach. Psychol., vol. 33, no. 4, pp. 236-239, Oct. 2006.

[13] D. A. Wilder, W. A. Flood, and W. Stromsnes, "The Use of Random Extra Credit Quizzes to Increase Student Attendance," J. Instr. Psychol. Milwaukee Wis, vol. 28, no. 2, pp. $117-$ 120, Jun. 2001. 